\title{
Bond Pricing with Jumps and Monte Carlo Simulation
}

\author{
Kisoeb Park ${ }^{1}$, Moonseong $\mathrm{Kim}^{2}$, and Seki Kim ${ }^{1, \star}$ \\ 1 Department of Mathematics, Sungkyunkwan University, \\ 440-746, Suwon, Korea \\ Tel.: +82-31-290-7030, 7034 \\ \{kisoeb, skim\}@skku.edu \\ 2 School of Information and Communication Engineering, \\ Sungkyunkwan University, \\ 440-746, Suwon, Korea \\ Tel.: +82-31-290-7226 \\ moonseong@ece.skku.ac.kr
}

\begin{abstract}
We derive a general form of the term structure of interest rates with jump. One-state models of Vasicek, CIR(Cox, Ingersol, and Ross), and the extended model of the Hull and White are introduced and the jump-diffusion models of the Ahn \& Thompson and the Baz \& Das as developed models are also investigated by using the Monte Carlo simulation which is one of the best methods in financial engineering to evaluate financial derivatives. We perform the Monte Carlo simulation with several scenarios even though it takes a long time to achieve highly precise estimates with the brute force method in terms of mean standard error which is one measure of the sharpness of the point estimates.
\end{abstract}

\section{Introduction}

We introduce one-state variable model of Vasicek [9], Cox, Ingersoll, and Ross (CIR) 3, the extended model of the Hull and White 6], and the development of the models which are the jump-diffusion model of the Ahn and Thompson [1] and the Baz and Das[2]. Conventionally, financial variables such as stock prices, foreign exchange rates, and interest rates are assumed to follow a diffusion processes with continuous paths when pricing financial assets.

In pricing and hedging with financial derivatives, jump-diffusion models are particularly important, since ignoring jumps in financial prices will cause pricing and hedging rates. For interest rates, jump-diffusion processes are particularly meaningful since the interest rate is an important economic variables which is, to some extent, controlled by the government as an instrument. Term structure model solutions under jump-diffusions are justified because movements in interest rates display both continuous and discontinuous behavior. These jumps are caused by several market phenomena money market interventions by the Fed, news surprise, and shocks in the foreign exchange markets, and so on.

\footnotetext{
^ Corresponding author.
} 
Ahn and Thompson[1] extended the CIR model by adding a jump component to the square root interest rate process. Using linearization technique, they obtained closed-form approximations for discount bond prices. Also, Baz, and Das 2] extended the Vasicek model by adding a jump component to the OrnsteinUhlenbeck $(\mathrm{O}-\mathrm{U})$ interest rate process, and obtained closed form approximate solutions for bond price by the same linearization technique.

All of the models mentioned above take special functional forms for the coefficients of $d t, d W$, and $d \pi$ in the stochastic differential equation for $r$. We derive a general form of the term structure of interest rate with jump and study a solution of the bond pricing for the above models. As above present a model which allows the short term interest rate, the spot rate, the follow a random walk. This leads to a parabolic partial differential equation for the prices of bonds and to models for bonds and many other interest rate derivative products. Above in result, we look into as the Vasicek, the CIR, the Hull and White, and the jump-diffusion models.

In addition, we introduce the Monte Carlo simulation. One of the many uses of Monte Carlo simulation by financial engineers is to place a value on financial derivatives. Interest in use of Monte Carlo simulation for bond pricing is increasing because of the flexibility of the methods in handling complex financial instruments. One measure of the sharpness of the point estimate of the mean is Mean Standard Error(MSE). Numerical methods that are known as Monte Carlo methods can be loosely described as statistical simulation methods, where statistical simulation is defined in quite general terms to be any method that utilizes sequences of random numbers to perform the simulation.

The structure of the remainder of this paper is as follows. In Section 2, the basic of bond prices with jump are introduced. In Section 3, the term structure models with jump are presented. In Section 4, we calculate numerical solutions using Monte Carlo simulation for the term structure models with jump. In Section 5, we investigate bond prices given for the eight models using the Vasicek and the CIR models. This paper is finally concluded in Section 6 .

\section{Bond Pricing Equation with Jump}

In view of our uncertainty about the future course of the interest rate, it is natural to model it as a random variable. To be technically correct we should specify that $r$ is the interest rate received by the shortest possible deposit. The interest rate for the shortest possible deposit is commonly called the spot rate. In the same way that a model for the asset price is proposed as a lognormal random walk, let us suppose that the interest rate $r$ is governed by a stochastic differential equation(SDE) of the form

$$
d r=u(r, t) d t+\omega(r, t) d W+J d \pi
$$

The functional forms of $\omega(r, t), u(r, t)$ (the instantaneous volatility and the instantaneous drift, respectively), and jump size $J$ is normal variable with mean $\mu$ 
and standard deviation $\gamma$ determine the behavior of the spot rate $r$. We consider a one-dimensional jump-diffusion process $r(t)$ is satisfying

$$
r(t)=r(0)+\int_{0}^{t} u(r, s) d s+\int_{0}^{t} \omega(r, s) d W(s)+\sum_{i=1}^{\pi(t)} J_{i},
$$

where $\pi(t)$ represents the number of jumps happening during the period between time 0 and $t$. When interest rates follow the $\mathbf{S D E}(1)$, a bond has a price of the form $V(r, t)$; the dependence on $T$ will only be made explicit when necessary. We set up a riskless portfolio and the jump-diffusion version of Ito's lemma to functions of $r$ and $t$. And then, we derive the partial differential bond pricing equation.

Theorem 1. If $r$ satisfy Stochastic differential equation $d r=u(r, t) d t+\omega(r, t)$ $d W+J d \pi$ then the zero-coupon bond pricing equation in jumps is

$$
\frac{\partial V}{\partial t}+\frac{1}{2} \omega^{2} \frac{\partial^{2} V}{\partial r^{2}}+(u-\lambda \omega) \frac{\partial V}{\partial r}-r V+h E[V(r+J, t)-V(r, t)]=0,
$$

where $\lambda(r, t)$ is the market price of risk. The final condition corresponds to the payoff on maturity and so $V(r, T, T)=1$. Boundary conditions depend on the form of $u(r, t)$ and $\omega(r, t)$.

\section{Term Structure Models with Jump}

We denote by $V(r, r, T)$ the price at time $t$ of a discount bond. It follows immediately that $V(r, T, T)=1$. In our framework, the yield curve is the same as term structure of interest rate, as we only work with zero-coupon bonds. Now consider a quite different type of random environment. Suppose $\pi(t)$ represents the total number of extreme shocks that occur in a financial market until time $t$. The time dependence can arise from the cyclical nature of the economy, expectations concerning the future impact of monetary policies, and expected trends in other macroeconomic variables. In this study, we extend the jump-diffusion version of equilibrium single factor model to reflect this time dependence. This leads to the following model for $r$ :

$$
d r(t)=[\theta(t)-a(t) r(t)] d t+\sigma(t) r(t)^{\beta} d W(t)+J d \pi(t)
$$

where $\theta(t)$ is a time-dependent drift; $\sigma(t)$ is the volatility factor; $a(t)$ is the reversion rate. We investigate the $\beta=0$ case is an extension of Vasicek's jumpdiffusion model; the $\beta=0.5$ case is an extension of CIR jump-diffusion model.

\subsection{Jump-Diffusion Version of Extended Vasicek's Model}

We proposed the mean reverting process for interest rate $r$ is given by the equa$\operatorname{tion}(4)$ with $\beta=0$ :

$$
d r(t)=[\theta(t)-a(t) r(t)] d t+\sigma(t) d W(t)+J d \pi(t)
$$


We will assume that the market price of interest rate diffusion risk is a function of time, $\lambda(t)$. Let us assume that jump risk is diversifiable. From equation (5) with the drift coefficient $u(r, t)=\theta(t)-a(t) r(t)$ and the volatility coefficient $\omega(r, t)=\sigma(t)$, we get the partial differential difference bond pricing equation:

$$
\begin{aligned}
{[\theta(t)-a(t) r(t)} & -\lambda(t) \sigma(t)] V_{r}+V_{t}+\frac{1}{2} \sigma(t)^{2} V_{r r}-r V \\
& +h V\left[-\mu A(t, T)+\frac{1}{2}\left(\gamma^{2}+\mu^{2}\right) A(t, T)^{2}\right]=0 .
\end{aligned}
$$

The price of a discount bond that pays off $\$ 1$ at time $T$ is the solution to (6) that satisfies the boundary condition $V(r, T, T)=1$. A solution of the form:

$$
V(r, t, T)=\exp [-A(t, T) r+B(t, T)]
$$

can be guessed. Bond price derivatives can be calculated from (7). We omit the details, but the substitution of this derivatives into (6) and equating powers of $\mathrm{r}$ yields the following equations for $A$ and $B$.

\section{Theorem 2}

$$
-\frac{\partial A}{\partial t}+a(t) A-1=0
$$

and

$$
\frac{\partial B}{\partial t}-\phi(t) A+\frac{1}{2} \sigma(t)^{2} A^{2}+h\left[-\mu A+\frac{1}{2}\left(\gamma^{2}+\mu^{2}\right) A^{2}\right]=0,
$$

where, $\phi(t)=\theta(t)-\lambda(t) \sigma(t)$ and all coefficients is constants. In order to satisfy the final data that $V(r, T, T)=1$ we must have $A(T, T)=0$ and $B(T, T)=0$.

\subsection{Jump-Diffusion Version of Extended CIR Model}

We propose the mean reverting process for interest rate $r$ is given by the equa$\operatorname{tion}(4)$ with $\beta=0.5$ :

$$
d r(t)=[\theta(t)-a(t) r(t)] d t+\sigma(t) \sqrt{r(t)} d W(t)+J d \pi(t)
$$

We will assume that the market price of interest rate diffusion risk is a function of time, $\lambda(t) \sqrt{r(t)}$. Let us assume that jump risk is diversifiable.

In jump-diffusion version of extended Vasicek's model the short-term interest rate, $r$, to be negative. If Jump-diffusion version of extended CIR model is proposed, then rates are always non-negative. This has the same mean-reverting drift as jump-diffusion version of extended Vasicek's model, but the standard deviation is proportional to $\sqrt{r(t)}$. This means that its standard deviation increases when the short-term interest rate increases. From equation $(3)$ with the drift coefficient $u(r, t)=\theta(t)-a(t) r(t)$ and the volatility coefficient $\omega(r, t)=\sigma(t) \sqrt{r(t)}$, we get the partial differential bond pricing equation: 


$$
\begin{aligned}
{[\theta(t)-a(t) r(t)} & -\lambda(t) \sigma(t) r(t)] V_{r}+V_{t}+\frac{1}{2} \sigma(t)^{2} r(t) V_{r r}-r V \\
& +h V\left[-\mu A(t, T)+\frac{1}{2}\left(\gamma^{2}+\mu^{2}\right) A(t, T)^{2}\right]=0 .
\end{aligned}
$$

Bond price partial derivatives can be calculated from (11). We omit the details, but the substitution of this derivatives into (7) and equating powers of $\mathrm{r}$ yields the following equations for $A$ and $B$.

\section{Theorem 3}

$$
-\frac{\partial A}{\partial t}+\psi(t) A+\frac{1}{2} \sigma(t)^{2} A^{2}-1=0
$$

and

$$
\frac{\partial B}{\partial t}-(\theta(t)+h \mu) A+\frac{1}{2} h\left[\left(\gamma^{2}+\mu^{2}\right) A^{2}\right]=0,
$$

where, $\psi(t)=a(t)+\lambda(t) \sigma(t)$ and all coefficients is constants. In order to satisfy the final data that $V(r, T, T)=1$ we must have $A(T, T)=0$ and $B(T, T)=0$.

Proof). In equations (12) and (13), by using the solution of this Ricatti's equation formula we have

$$
A(t, T)=\frac{2\left(e^{\omega(t)(T-t)}-1\right)}{(\omega(t)+\psi(t))\left(e^{\omega(t)(T-t)}-1\right)+2 \omega(t)}
$$

with $\omega(t)=\sqrt{\psi(t)^{2}+2 \sigma(t)}$. Similarly way, we have

$$
B(t, T)=\int_{t}^{T}\left\{-(\theta(t)+h \mu) A+\frac{1}{2} h\left(\gamma^{2}+\mu^{2}\right) A^{2}\right\} d t^{\prime} .
$$

These equation yields the exact bond prices in the problem at hand. Equation (15) can be solved numerically for $B$. Since (14) gives the value for $A$, bond prices immediately follow from equation (7).

\section{Monte Carlo Simulation of the Term Structure Models with Jump}

Recent methods of bond pricing do not necessarily exploit partial differential equations(PDEs) implied by risk-neutral portfolios. They rest on converting prices of such assets into martingales. This is done through transforming the underlying probability distribution using the tools provided by the Girsanov's theorem. We now move on to discuss Monte Carlo simulation. A Monte Carlo simulation of a stochastic process is a procedure for sampling random outcomes for the process. This uses the risk-neutral valuation result. The bond price can be expressed as:

$$
V\left(r_{t}, t, T\right)=E_{t}^{Q}\left[e^{-\int_{t}^{T} r_{s} d s} \mid r(t)\right]
$$


where $E^{Q}$ is the expectations operator with respect to the equivalent risk-neutral measure. To execute the Monte Carlo simulation, we discretize the equations (5) and (12). we divide the time interval $[t, T]$ into $m$ equal time steps of length $\Delta t$ each. For small time steps, we are entitled to use the discretized version of the risk-adjusted stochastic differential equations (5) and (12):

$$
\begin{aligned}
r_{j} & =r_{j-1}+\left[(\theta \cdot t)-(a \cdot t) r_{j-1} \cdot t-(\lambda \cdot t)(\sigma \cdot t)\right] \Delta t \\
& +(\sigma \cdot t) \varepsilon_{j} \sqrt{\Delta t}+J_{j} N_{\Delta t}
\end{aligned}
$$

and

$$
\begin{aligned}
r_{j} & =r_{j-1}+\left[(\theta \cdot t)-(a \cdot t) r_{j-1}-(\lambda \cdot t)(\sigma \cdot t) \sqrt{r_{j-1} \cdot t}\right] \Delta t \\
& +(\sigma \cdot t) \sqrt{r_{j-1} \cdot t} \varepsilon_{j} \sqrt{\Delta t}+J_{j} N_{\Delta t},
\end{aligned}
$$

where $j=1,2, \cdots, m, \varepsilon_{j}$ is standard normal variable with $\varepsilon_{j} \sim N(0,1)$, and $N_{\Delta t}$ is a Poisson random variable with parameter $h \Delta t$. We can investigate the value of the bond by sampling $n$ short rate paths under the discrete process approximation of the risk-adjusted processes of the equations (17) and (18). The bond price estimate is given by:

$$
V\left(r_{t}, t, T\right)=\frac{1}{n} \sum_{i=1}^{n} \exp \left(-\sum_{j=0}^{m-1} r_{i j} \Delta t\right),
$$

where $r_{i j}$ is the value of the short rate under the discrete risk-adjusted process within sample path $i$ at time $t+\Delta t$. Numerical methods that are known as Monte Carlo methods can be loosely described as statistical simulation methods, where statistical simulation is defined in quite general terms to be any method that utilizes sequences of random numbers to perform the simulation. The Monte Carlo simulation is clearly less efficient computationally than the numerical method. The precision of the mean as a point estimate is often defined as the half-width of a $95 \%$ confidence interval, which is calculated as

$$
\text { Precision }=1.96 \times M S E,
$$

where $\mathrm{MSE}=\nu / \sqrt{n}$ and $\nu^{2}$ is the estimate of the variance of bond prices as obtained from $n$ sample paths of the short rate:

$$
\nu^{2}=\frac{\sum_{i=1}^{n}\left[\exp \left(-\sum_{j=0}^{m-1} f_{i j} \Delta t\right)-\nu\right]}{n-1}
$$

Lower values of Precision in Equation(20) correspond to sharper estimates. Increasing the number of $n$ is a brute force method of obtaining sharper estimates. This reduces the MSE by increasing the value of $n$. However, highly precise estimates with the brute force method can take a long time to achieve. For the 
purpose of simulation, we conduct three runs of 1,000 trials each and divide the year into 365 time steps.

\section{Experiments}

In this section, we investigate the Vasicek, the CIR, the Hull and White, and jump diffusion version of three models. Experiments are consist of the numerical method and Monte Carlo simulation. Experiment 1, 2 plot estimated term structure using the four models. In Experiment 1 and 2, the parameter values are assumed to be $r=0.05, a=0.5, b=0.05, \theta=0.025, \sigma=0.08, \lambda=-0.5$, $\gamma=0.01, \mu=0, h=10, t=0.05$, and $T=20$.

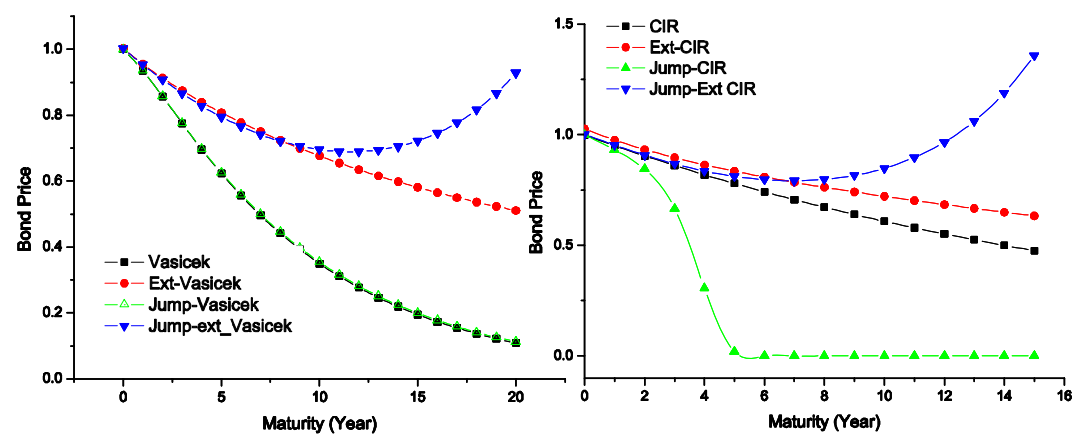

(a) Bond prices for the various Vasicek models

(b) Bond prices for the various CIR models

Fig. 1. Experiment 1 and 2: The various bond prices

Table 1. Experiment 3: Bond prices estimated by the Monte Carlo simulation for the Vasicek, the extended Vasicek, and the jump diffusion version of the Vasicek and the extended Vasicek, the CIR, the extended CIR, and the jump diffusion version of the CIR and the extended CIR models

\begin{tabular}{|c|c|c|c|c|c|}
\hline & CFS & MCS & CFS - MCS & Variance & Precision \\
\hline \hline Vasicek & 0.93585 & 0.93492 & 0.0013423 & 0.001159 & 0.00182868 \\
\hline Ext_Vasicek & 0.95408 & 0.95122 & $5.0085 \mathrm{E}-06$ & $7.0771 \mathrm{E}-05$ & 0.0056056 \\
\hline jump-Vasicek & 0.93596 & 0.93391 & 0.001508 & 0.001228 & 0.004018 \\
\hline jump-Ext_Vasice & 0.95370 & 0.95031 & 0.000287 & 0.000536 & 0.00665224 \\
\hline CIR & 0.95127 & 0.94747 & $7.2915 \mathrm{E}-05$ & 0.00027 & 0.0074284 \\
\hline Ext_CIR & 0.97604 & 0.95123 & $2.42154 \mathrm{E}-07$ & $1.5561 \mathrm{E}-05$ & 0.0486472 \\
\hline jump-CIR & 0.942005 & 0.947482 & 0.005478 & 0.000286321 & 0.0010488 \\
\hline jump-Ext_CIR & 0.95348 & 0.95169 & 0.0017904 & 0.000306335 & 0.00108482 \\
\hline
\end{tabular}

Experiment 3 examines bond prices using by Monte Carlo simulation. In experiment 3, the parameter values are assumed to be $r=0.05, a=0.5, b=0.05$, $\theta=0.025, \sigma=0.08, \lambda=-0.5, \Delta t=(T-t) / m, m=365, n=1000, \gamma=0.01$, $\mu=0, h=10, t=0.05$, and $T=20$. 


\section{Conclusions}

The Monte Carlo simulation is both harder and conceptually more difficult to implement than the other numerical methods. Interest in use of Monte Carlo simulation for bond pricing is getting stronger because of its flexibility in evaluating and handling complicated financial instruments. However, it takes a long time to achieve highly precise estimates with the brute force method. In this paper we investigate bond pricing models and their Monte Carlo simulations with several scenarios. The bond price is generally a decreasing function of the maturity, but we found the fact that the bond price is humped in the jump versions of the extended Vasicek and CIR models. In Monte Carlo simulation, we know that the bond prices of the arbitrage models are larger than those of the equilibrium models. Also lower values of precision in the equilibrium models correspond to sharper estimates.

\section{References}

1. C. Ahn and H. Thompson, "Jump-Diffusion Processes and the Term Structure of Interest Rates," Journal of Finance, vol. 43, pp. 155-174, 1998.

2. J. Baz and S. R. Das, "Analytical Approximations of the Term Structure for JumpDiffusion Processes : A Numerical Analysis," Journal of Fixed Income, vol. 6(1), pp. 78-86, 1996.

3. J. C. Cox, J. Ingersoll, and S. Ross, "A Theory of the Term Structure of Interest Rate," Econometrica, vol. 53, pp. 385-407, 1985.

4. D. Health, R. Jarrow, and A. Morton, "Bond Pricing and the Term Structure of Interest Rates," Econometrica, vol. 60. no. 1, pp. 77-105, 1992.

5. F. Jamshidian, "An Exact Bond Option Formula," Journal of Finance, vol. 44, 1989.

6. J. Hull and A. White, "Pricing Interest Rate Derivative Securities," Review of Financial Studies, vol. 3, pp. 573-92, 1990.

7. J. Hull and A. White, "Options, Futures, and Derivatives," Fourth Edition, 2000.

8. M. J. Brennan and E. S. Schwartz, "A Continuous Time Approach to the Pricing of Bonds," Journal of Banking and Finance, vol. 3, pp. 133-155, 1979.

9. O. A. Vasicek, "An Equilibrium Characterization of the Term Structure," Journal of Financial Economics, vol. 5, pp. 177-88, 1977. 